\title{
La transición del pensamiento de Wittgenstein y la posible influencia de los economistas de Cambridge
}

The Transition of Wittgenstein's Thought and the Potential Influence of Cambridge Economists

A transição do pensamento de Wittgenstein e a possível influência dos economistas de Cambridge

Germán Raúl Chaparro*

DOI: https://doi.org/10.19053/01203053.v38.n67.2019.8219

Fecha de recepción: 18 de noviembre de 2016

Fecha de aceptación: 25 de mayo de 2017

Cómo citar este artículo/ to reference this article / comment citer cet article / para citar este artigo:

Chaparro, G. (2019). La transición del pensamiento de Wittgenstein y la influencia de los economistas de Cambridge. Apuntes del Cenes, 38(67). https://doi.org/10.19053/01203053.v38.n67.2019.8219

* Magíster en Economía y estudiante de Doctorado en Historia. Investigador del Grupo de Protección Social de la Universidad Nacional de Colombia y profesor asistente de la Maestría en Tributación de la Universidad Central. Bogotá, Colombia. rchaparrog@unal.edu.co (10 https://orcid.org/0000-0002-6443-0021. 


\section{Resumen}

El artículo tiene por objeto revisar la posible influencia de John Maynard Keynes, Frank Ramsey, Piero Sraffa y, por intermedio de este último, Antonio Gramsci, sobre la transición del pensamiento filosófico de Ludwig Wittgenstein. El trabajo reconstruye, a partir de la literatura especializada, la relación del filósofo austriaco con los economistas que conoció en Cambridge y propone que estos pudieron haber permeado el quehacer filosófico de Wittgenstein y, en particular, la forma en que la perspectiva antropológica de los problemas filosóficos, posiblemente inspirada en Gramsci y transmitida por Sraffa, fue recibida y desarrollada por Wittgenstein en sus Investigaciones Filosóficas, en el desarrollo de conceptos como las formas de vida y los juegos del lenguaje. Se concluye que las teorías de Gramsci sobre la hegemonía y la alienación lingüística tienen cierta similitud con la propuesta de filosofía del lenguaje cotidiano de Wittgenstein.

Palabras clave: lenguaje cotidiano, filosofía política, economía, historia, pensamiento económico.

Clasificación JEL: A11, A12, B30, B41, B50, B51. 


\section{Abstract}

The aim of this paper is to review the possible influence of John Maynard Keynes, Frank Ramsey, Piero Sraffa and, through the latter, Antonio Gramsci, on the transition of the philosophical thought of Ludwig Wittgenstein. The work reconstructs, from specialized literature, the relationship of the Austrian philosopher with the economists he met at Cambridge and proposes that these may have permeated the philosophical work of Wittgenstein and, in particular, the way in which the anthropological perspective of the philosophical problems, possibly inspired by Gramsci and transmitted by Sraffa, was received and developed by Wittgenstein in his Philosophical Investigations, in the development of concepts such as form of life and language games. It is concluded that Gramsci's theories about hegemony and linguistic alienation bear a certain similarity to Wittgenstein's philosophy of everyday language.

Keywords: everyday language, political philosophy, economy, history, economic thought. 


\section{Resumo}

O artigo tem como objetivo revisar a possível influência do John Maynard Keynes, Frank Ramsey, Piero Sraffa e, através deste último, Antonio Gramsci sobre a transição do pensamento filosófico de Ludwig Wittgenstein. O trabalho reconstrói, a partir da literatura especializada, a relação do filósofo austríaco com os economistas que conheceu em Cambridge e propõe que estes possam ter permeado o trabalho filosófico de Wittgenstein e, em particular, a maneira pela qual a perspectiva antropológica dos problemas Filosófico, possivelmente inspirado por Gramsci e transmitido por Sraffa, foi recebido e desenvolvido por Wittgenstein em suas Investigações Filosóficas, no desenvolvimento de conceitos como formas de vida e jogos da linguagem. Conclui-se que as teorias do Gramsci sobre a hegemonia e a alienação linguística apresentam certa semelhança com a filosofia da linguagem cotidiana de Wittgenstein.

Palavras-chave: linguagem cotidiana, filosofia política, economia, história, pensamento econômico. 


\section{INTRODUCCIÓN:}

\section{EL REGRESO DE UN "ÁNGEL"}

"Bueno, Dios ha llegado. Le encontré en el tren de las 5:15". Estas fueron las palabras de John Maynard Keynes a su esposa, Lidia Lopokova, en las que anunciaba que, finalmente, el 18 de enero de 1929, Ludwig Josef Johann Wittgenstein (1889-1951) había regresado a Cambridge para quedarse de manera permanente ${ }^{1}$. En la misma comunicación señala: "Veo que a partir de ahora la fatiga será abrumadora. No debo dejar que me hable durante más de dos o tres horas al día". Wittgenstein era para entonces una leyenda en
Cambridge, su Tractatus logicophilosophicus, publicado en 1922, había sido de gran influencia sobre los positivistas lógicos del Círculo de Viena y estaba en el centro de casi toda la discusión intelectual de la época.

El Tractatus era producto de las reflexiones de Wittgenstein durante su primera estancia en Cambridge entre 1912 y 1913 , donde contó con la asesoría de Bertrand Russell ${ }^{2}$, aunque buena parte de las notas que lo componen fueron manuscritas en las trincheras donde combatió durante la Primera Guerra Mundial. En efecto, Wittgenstein se alistó como voluntario

1 Carta de Keynes a Lidia Lopokova (Wright, 2001, p. 243), citada también por Dostaler (2007, p. 63) y Monk (1994, p. 243).

2 Bertrand Russell y Alfred North Whitehead acababan de publicar los Principia mathematica. Las inquietudes de Wittgenstein en esa época eran las cuestiones de lógica planteadas por estos autores y por Gottlob Frege. En el Trinity College, Wittgenstein tomó cursos de lógica con W. E. Johnson y con G. E. Moore.

Entonces Russell describía a Wittgenstein como: "quizá el más perfecto ejemplo que he conocido jamás de un genio tal como se concibe tradicionalmente, apasionado, profundo, intenso y dominante" (Monk, 1994, p. 58). Una mente rigurosamente lógica y de una naturaleza impulsiva y obsesiva que pronto llamó la atención de los Apóstoles, un grupo de debate del cual Russell era miembro, y que estaba dominado por Keynes y Lytton Strachey. 
en el ejército austriaco, aunque había sido eximido a causa de una hernia. Primero luchó en el frente este y, luego, en 1918, fue trasladado al frente sur, allí fue puesto prisionero por el ejército italiano. Cuando fue capturado llevaba en su mochila un manuscrito titulado Logisch-philosophische Abhandlung, que después, por sugerencia de G. E. Moore, tomaría el título latino de Tractatus Logico-Philosophicus (Wright, 2001, p. 9). En el Tractatus, Wittgenstein presenta una teoría figurativa del lenguaje, la cual supone la existencia de una correspondencia entre el mundo y el lenguaje. La idea central consiste en que toda proposición del lenguaje debe estar asociada a un estado de cosas efectivo (conexiones o relaciones entre cosas u objetos) del mundo, es decir, una proposición es la imagen de un estado de cosas que retrata, por eso una proposición y lo que ella describe deben compartir una estructura común, la misma forma lógica. Esto permite la representación de la realidad por medio del lenguaje.

Una anécdota contada por Wittgenstein a Norman Malcolm sobre el origen de la idea central de Tractatus resulta ilustrativa:

Esta idea se le ocurrió a Wittgenstein mientras servía en el ejército austríaco durante la Primera Guerra Mundial.
Vio un periódico que describía la ocurrencia y localización de un accidente automovilístico por medio de un diagrama o mapa. Se le ocurrió entonces a Wittgenstein que este mapa era una proposición y que en ella se revelaba la naturaleza esencial de las proposiciones, a saber, el describir la realidad. (Malcolm, 2001, p. 57)

Al retornar al círculo intelectual de Cambridge, Wittgenstein se reencontró, además de Keynes, con antiguos conocidos como George Edward Moore y Bertrand Russell; y conocería a varios personajes que se destacaban en el terreno de la teoría económica, en particular a Frank Ramsey y Piero Sraffa. La segunda estancia en la Universidad de Cambridge implicó una transformación de la visión filosófica de Wittgenstein, revaluó las posturas del positivismo lógico y desarrolló una teoría de los juegos del lenguaje, en la que propone entender el lenguaje como producto de una práctica social (Peña, 1994, p. 23-24).

La relación de Wittgenstein con algunos de los economistas de Cambridge parece haber ejercido cierta influencia en su tránsito hacia una segunda filosofía. De hecho, en el prefacio de las Philosophische Untersuchungen (Investigaciones filosóficas) ${ }^{3}$, Wittgenstein agradece la influencia recibida por

3 Libro póstumo editado por Elizabeth Anscombe y publicado en 1953. 
parte de Ramsey y Sraffa, en particular señala que la crítica de este último constituyó el más importante estímulo a su trabajo:

A advertir estos errores me ha ayudado - en un grado que apenas yo mismo puedo apreciar - la crítica que mis ideas han encontrado en Frank Ramsey, — con quien las he discutido durante los dos últimos años de su vida en innumerables conversaciones-. Más aún que a esta crítica — siempre potente y certera - le debo a la que un profesor de esta universidad, el señor P. Sraffa, ha practicado durante muchos años sin interrupción sobre mis pensamientos. A este aguijón le debo las ideas más ricas en consecuencias de este escrito. (Wittgenstein, 2009, p. 163)

Wittgenstein y Sraffa mantuvieron discusiones semanales que se extendieron desde principios de la década que inició en 1930 hasta 1946, cuando el economista italiano decidió poner fin a esos encuentros. La posible influencia de Ramsey también debió ser importante si consideramos, además, que este colaboró en la realización de la primera traducción del Tractatus al inglés y escribió una reseña crítica del mismo libro, además fue instructor de Wittgenstein durante su segunda estadía en Cambridge.
El presente artículo revisa la posible influencia del círculo de economistas de Cambridge en el tránsito de Wittgenstein hacia su segunda filosofía. En particular, se discute la influencia de Piero Sraffa y la llamada "conexión gramsciana", esto es, la influencia indirecta, mediada por Sraffa, de Antonio Gramsci sobre Wittgenstein. El artículo se divide en cuatro secciones: en la primera se revisa la relación de Wittgenstein con Keynes y Ramsey; en la segunda, la amistad con Sraffa; y en la tercera, la posible influencia indirecta de Gramsci. En la última sección se presentan las conclusiones.

\section{WITTGENSTEIN VUELVE A CAMBRIDGE: LA INFLUENCIA DE KEYNES Y RAMSEY}

Wittgenstein terminó de escribir el Tractatus en 1918 y tenía la convicción de haber resuelto todos los problemas de la lógica filosófica que se había planteado, como lo deja ver el prólogo que escribió en Viena:

El libro trata los problemas filosóficos y muestra -según creo- que el planteamiento de estos problemas descansa en la incomprensión de la lógica de nuestro lenguaje. [...] $\mathrm{La}$ verdad de los planteamientos aquí comunicados me parece, en cambio, intocable y definitiva. Soy, pues, de la opinión de haber solucionado definitivamente, en lo esencial, los 
problemas. (Wittgenstein, 2015, pp. 55-56)

En consecuencia, Wittgenstein tomó entonces la decisión prematura de abandonar la filosofia (Wright, 2001, p. 11).

Finalizada la Gran Guerra decidió renunciar a la fortuna heredada de su padre, un industrial exitoso que logró acumular una de las mayores fortunas de Viena, y trabajar como profesor de escuela, con la intención idealista de incrementar el nivel intelectual de los niños de la Austria rural y menos favorecida económicamente, al tiempo que podría llevar una vida austera, cercana al ascetismo. De esta manera, durante los años siguientes Wittgenstein fue profesor en los pueblos de Trattenbach, Hassbach y Puchberg.

En Trattenbach se puso a la tarea de escribir un diccionario, el Wörterbuch für Volksschulen (publicado en Viena en 1926), con el objetivo de enseñar a los niños ortografía y gramática a partir de la lengua hablada corriente, incluidos los dialectos (Monk, 1994, pp. 217-218). Bouveresse ha señalado que:

Es probable que los problemas concretos de pedagogía y de psicología con los que Wittgenstein forzosamente se encontró en contacto directo durante esos años, hayan tenido, en la génesis de lo que se ha convenido llamar su 'segunda filosofía', un papel al menos tan importante como el que él le atribuyó a la influencia de hombres como Ramsey y Sraffa. (Bouveresse, 2006, p. 68)

Dos economistas de Cambridge, John Maynard Keynes y Frank Plumpton Ramsey, serían determinantes en que Wittgenstein decidiera volver a la filosofía. Keynes, quien lo conocía desde su primera estancia en Cambridge, lo ayudó en varias ocasiones, por ejemplo, cuando Wittgenstein estuvo prisionero en Italia, en 1919, le ayudó a gestionar la correspondencia, con lo cual el manuscrito del Tractatus pudo llegar a manos de Russell y Frege; promovió su regreso a Cambridge en 1929; lo recomendó como sucesor de la cátedra de Filosofía Moral de Moore en 1939, y también en el proceso de obtener la ciudadanía británica justo antes de que estallara la Segunda Guerra mundial.

Es probable que el análisis de la importancia de las reglas y de las convenciones en la toma de decisiones de inversión, desarrolladas por Keynes en la Teoría general de la ocupación, el interés y el dinero, tuviera alguna influencia en la segunda filosofía de Wittgenstein, pues en las Investigaciones Filosóficas se reconoce que las convenciones sociales dan sentido 
y significado a las expresiones del lenguaje. Por su parte, Wittgenstein criticó el Tratado de probabilidad de Keynes, pero mostró una mejor apreciación de trabajos como Las consecuencias económicas de la paz, el cual consideraba de "enorme importancia" e influencia (McGuinness, 2008, p. 115), y de una pequeña revisión de la situación de Rusia (A Short View of Russia) (McGuinness, 2008, p. 162).

El economista británico no era ajeno al mundo de la filosofía, de hecho, su disertación para obtener la titularidad como profesor del King's College abordaba la filosofía de la probabilidad, lo que después, en 1921, se convirtió en un libro: A Treatise on Probability (Backhouse \& Bateman, 2014, p. 29). Sin embargo, Keynes reconoció la dificultad de la lectura del Tractatus, tras un intento fallido de explicarlo al grupo de los Apóstoles, en una carta a Lydia Lopokova (15 de noviembre de 1925) señaló: "Anoche traté de explicar la filosofia de Ludwig a mi Sociedad, pero ella escapa a la mente -sólo podía recordarla a medias" (Dostaler, 2007, p. 64).

Entre 1921 y 1922, Bertrand Russell se encargó de la publicación del Tractatus en Inglaterra y en Alemania. El libro, escrito originalmente en alemán, fue traducido al inglés por Frank Ramsey, en ese momento un estudiante de 18 años del King College y reconocido como una promesa en el campo de las matemáticas. Ramsey escribió una reseña del Tractatus en la que criticaba el atomismo lógico y la idea de un lenguaje lógicamente perfecto propuesta por Wittgenstein, además señalaba la dificultad de explicar la incompatibilidad de colores como una contradicción:

Este es un principio de la teoría del Sr. Wittgenstein y, si fuera verdadero, sería un descubrimiento muy importante, que toda proposición genuina afirma algo posible, pero no necesario. Esto se sigue de su tratamiento de una proposición como la expresión de acuerdo y desacuerdo, con las posibilidades de verdad de las proposiciones elementales independientes, de tal modo que la única necesidad es la de la tautología, la única imposibilidad la de la contradicción. Hay una gran dificultad para sostener esto; porque el Sr. Wittgenstein admite que un punto en el campo visual no puede ser a la vez rojo y azul; y de hecho, en el caso contrario, puesto que piensa que la inducción no tiene bases lógicas, no deberíamos tener razón para pensar que no podemos encontrarnos con un punto visual que sea a la vez rojo y azul. De aquí que él diga que 'Esto es a la vez rojo y azul' es una contradicción. Esto implica que los conceptos, aparentemente simples, de rojo y azul (suponiendo que lo 
que queremos decir con estas palabras son tonalidades absolutamente específicas) son realmente complejos y formalmente incompatibles. Él trata de mostrar cómo puede ocurrir esto analizándolos en términos de vibraciones. Pero incluso suponiendo que el físico proporcionará un análisis de lo que queremos decir con "rojo", el Sr. Wittgenstein solo está reduciendo la dificultad a la de las propiedades necesarias de espacio, tiempo y materia, o el éter. Explícitamente lo hace depender de la imposibilidad de que una partícula esté en dos lugares al mismo tiempo. Estas propiedades necesarias de espacio y tiempo difícilmente son susceptibles de una reducción ulterior de este tipo. Por ejemplo, considerando el estar entre en un momento del tiempo por lo que toca a mis experiencias; si $\mathrm{B}$ está entre A y $\mathrm{D}, \mathrm{y} \mathrm{C}$ entre $\mathrm{B}$ y $\mathrm{D}$, entonces $\mathrm{C}$ debe estar entre $\mathrm{A}$ y $\mathrm{D}$; pero es difícil ver cómo puede ser esto una tautología formal. (Ramsey, 1923, p. $473)^{4}$

Así, la incompatibilidad de colores es atribuida por Wittgenstein a las propiedades del espacio, del tiempo y de la materia y no se explica por la forma general de la proposición ${ }^{5}$.

Ramsey visitó a Wittgenstein en Puchberg en 1923, durante un par de semanas se reunieron para discutir el Tractatus, de allí salieron correcciones y cambios que fueron incluidos en las ediciones posteriores. Keynes, por intermedio de Ramsey, trató de atraer a Wittgenstein a Inglaterra, para que reanudara su trabajo filosófico en Cambridge (Monk, 1994, pp. 211215). Wittgenstein no aceptó la invitación de Keynes, según aquel porque no se sentía capacitado para volver a la filosofía, en una carta del 4 julio de 1924, dirigida a Keynes, decía: "ya no poseo el impulso interior hacia ese tipo de actividad. En realidad, todo lo que tenía que decir, ya lo he dicho, y la fuente se ha secado". Y añadía que, si tuviera algún empleo en Inglaterra, aunque fuera barrer las calles o limpiar botas, "iría con gran placer" (McGuinness, 2008, pp. 152-153).

\section{LA ANÉCDOTA DEL TREN Y EL POSIBLE INFLUJO DE PIERO SRAFFA}

Piero Sraffa (1898-1983) es uno de los economistas más importantes del

\footnotetext{
4 La obra filosófica completa de Ramsey, incluida la reseña al Tractatus, se encuentra traducida al español (Ramsey, 2005).

5 El artículo de Wittgenstein, Some Remarks on Logical Form, escrito en 1929, es un intento de responder a la crítica de Ramsey (Wright, 2001, p. 13). Se suponía que el artículo fuera leído en la reunión anual de filósofos británicos en 1929. Sin embargo, en la reunión Wittgenstein decidió hablar de un tema enteramente diferente: la noción del infinito en matemáticas; aparentemente pensaba que su informe original carecía de valor (Wright, 2001, p. 13).
} 
siglo XX, trabajó en una tradición heterodoxa desde la que buscó mostrar las debilidades de la economía marginalista y recuperar el enfoque clásico de Adam Smith, David Ricardo y Karl Marx (Roncaglia, 2006, p. 569). Su obra no parece muy extensa, consiste en algunos artículos, la edición de las obras completas de David Ricardo y el libro Producción de mercancías por medio de mercancías. Preludio a una crítica de la teoría económica (Sraffa, 1983), obra que ha tenido una influencia significativa en el desarrollo de la ciencia económica.

En la tesis doctoral: L'inflazione monetaria in Italia durante e dopo la guerra, presentada en 1920, Sraffa toma distancia de la teoría cuantitativa del dinero y muestra que el índice general de precios es una medida engañosa que oscurece el papel central de los conflictos sociales en la vida económica, pues desconoce los distintos puntos de vista de los trabajadores y los empresarios al respecto (Roncaglia, 2006, pp. 570571). En 1922 publicó en Inglaterra dos artículos sobre la crisis bancaria italiana; en el primero, publicado en el Economic Journal, analiza el atraso de los mercados financieros en Italia, la consecuente inevitabilidad de las relaciones directas entre bancos y empresas, los peligros derivados de estas relaciones y la dificultad de lograr un cambio en la situación debido a una falta de voluntad política real; en el segundo artículo, redactado a solicitud de Keynes y publicado en el Manchester Guardian, formula sus dudas sobre la corrección de la contabilidad oficial de los principales bancos comerciales y sobre la estrategia institucional adoptada para que estos bancos recibieran el apoyo de los bancos emisores eludiendo los límites legales (Roncaglia, 2006, p. 572). La publicación de estos artículos provocó una reacción áspera por parte de Benito Mussolini, por lo que Keynes facilitó la salida de Sraffa de Italia invitándolo como profesor a Cambridge en 1927, dos años antes del retorno de Wittgenstein.

Piero Sraffa y Ludwig Wittgenstein se conocieron a través de Keynes en 1929, y pronto se hicieron amigos y establecieron conversaciones regulares cada semana. Las preocupaciones de Sraffa estaban en el terreno de la economía, más que en el campo de la filosofía o de las matemáticas, por ello su contribución al pensamiento de Wittgenstein difícilmente puede estar en los detalles del razonamiento filosófico; no obstante, el inicio de las conversaciones con Sraffa coinciden con un cambio en la línea de argumentación de Wittgenstein, la cual se fue alejando de la tradición filosófica de Frege y Russell. Algo en esas conversaciones con Sraffa resultó estimulante para que Wittgenstein llegara a "reconocer graves errores" contenidos en el Tractatus y para renovar su filosofía (Wittgenstein, 2009, p. 163). Sraffa 
tenía el poder de obligar a Wittgenstein a revisar toda su perspectiva; Wittgenstein mismo dijo que las conversaciones con Sraffa le hicieron sentir como "un árbol al cual le han cortado todas sus ramas"; que este árbol pudiera reverdecer sería gracias a su propia vitalidad (Wright, 2001, pp. 14-15). "La metáfora está cuidadosamente elegida: podar las ramas muertas permite que crezcan otras nuevas y vigorosas (mientras que las objeciones de Ramsey dejaban la madera muerta sin podar obligando al árbol a deformarse)" (Monk, 1994, p. 248; Sen, 2003, p. 1242).

No es posible trazar de manera precisa la influencia de Sraffa sobre Wittgenstein, ni en su magnitud ni en su dirección. Se sabe que en mayo de 1946 Piero Sraffa decidió poner fin a las conversaciones con Wittgenstein (Monk, 1994, p. 443) y no escribió nada sobre sus razones o el contenido de esos diálogos; por su parte, el filósofo austriaco tampoco fue explícito en señalar los temas concretos o la naturaleza de la posible influencia que sobre él tuvo la crítica de Sraffa. Quizás por ello las conjeturas sobre tal influencia se suelen remitir a la conocida anécdota del tren:

Un día (creo que estaban viajando en un tren), cuando Wittgenstein estaba insistiendo en que una proposición y lo que ella describe debían tener la misma 'forma lógica', la misma 'multiplicidad lógica', Sraffa hizo un gesto, familiar para los napolitanos, que significa algo así como disgusto o desprecio, y que consiste en cepillar la parte inferior de la barbilla con un movimiento de barrido hacia fuera con las puntas de los dedos de una mano. Y entonces preguntó: ‘¿Cuál es la forma lógica de esto?'. El ejemplo de Sraffa produjo en Wittgenstein el sentimiento de que era un absurdo la insistencia en que una proposición y lo que ella describe deben tener la misma 'forma'. Esto rompió con su convicción en la concepción de que una proposición debe ser literalmente una 'imagen' de la realidad que ella describe. (Malcolm, 2001, pp. 57-58)

G. H. von Wright relata el incidente del tren de una manera diferente, sin aludir a las expresiones "forma lógica" o "multiplicidad lógica". Al parecer Wittgenstein habría planteado en la conversación la cuestión de si cada proposición debería tener una "gramática" (Malcolm, 2001, p. 58), ante lo cual Sraffa interpeló realizando el gesto napolitano y preguntando a su interlocutor: “¿Cuál es la gramática de esto?” (McGuinness, 2008, p. 9). En cualquier caso, la importancia del episodio radica en que Sraffa, por medio del gesto napolitano, pudo haber persuadido a Wittgenstein de que una proposición y lo que ella describe deben ser analizadas en el contexto de su uso. 
Amartya Sen, quien fue estudiante y luego colega de Sraffa en el Trinity College, le preguntó a Sraffa por este episodio, sin embargo, este nunca confirmó su ocurrencia; a partir de ahí se generó la idea de que se trataba de una historia apócrifa (Sen, 2003, p. 1242). Sin embargo, en una conversación con Alessandro Roncaglia en 1972, Sraffa confirmó la anécdota y afirmó que Von Wright tenía razón (Roncaglia, 2006, p. 585).

La anécdota del tren ilustra gráficamente la naturaleza del escepticismo de Sraffa respecto a la filosofía trazada en el Tractatus y, en particular, su convicción de que las convenciones sociales pueden contribuir al significado de nuestras expresiones y gestos (Sen, 2003, p. 1242). En este sentido, Wittgenstein le comentó a Rush Rhees que el principal aporte de Sraffa en las conversaciones fue proporcionarle una "óptica 'antropológica' para ver los problemas filosóficos" (Monk, 1994, p. 248). La segunda filosofía de Wittgenstein incorpora el análisis de las reglas y las convenciones, reconoce la dificultad de abordar el lenguaje de manera independiente de las circunstancias en que se utiliza, el flujo de vida es lo que da significado a las manifestaciones lingüísticas: "un 'juego de lenguaje' no puede describirse sin mencionar las actividades y el modo de vida de la 'tribu' que lo juega" (Monk, 1994, p. 248); así, el sentido y el significado de una expresión lingüística están determinados por el contexto de su uso, perspectiva que permite desarrollar una 'filosofía del lenguaje ordinario"” (Sen, 2003, p. 1242).

K. T. Fann ha propuesto que la crítica implícita en la anécdota del tren no constituye un contraejemplo decisivo, pues, según el Tractatus, el gesto no constituye una proposición, pues al ser un movimiento que expresa afectos o emociones no describe un hecho concreto; sin embargo sería importante por cuestionar la concepción de Wittgenstein de funcionamiento del lenguaje en una sola vía. Sraffa, según Fann, usa en Producción de mercancías por medio de mercancías un "método de antropología especulativa", procedimiento que consiste en partir de considerar una sociedad imaginaria y extremadamente simple a partir de la cual, mediante la adición gradual de características nuevas, se construyen formas cada vez más complejas; este método también sería crucial en la transición del pensamiento de Wittgenstein y encontraría su expresión en el intento del filósofo austriaco de imaginar y construir juegos del lenguaje, que primero son simples y luego van ganando complejidad mediante la adición de formas del lenguaje más complejas (Fann, 1975, pp. 69-70). 


\section{LA INFLUENCIA INDIRECTA DE ANTONIO GRAMSCI}

No se conoce la razón por la cual Sraffa decidió poner fin a las conversaciones regulares con Wittgenstein. Es posible que haya sido por la ingenuidad política de este, que se haya cansado del carácter dominante de su interlocutor - quien tenía fama de convertir las conversaciones en un monólogo-, o simplemente porque no consideraba provechosas estas conversaciones. Sen (2003) ha insistido en que lo que para Wittgenstein era un conocimiento nuevo, para Sraffa era una obviedad, quizás porque en los círculos intelectuales italianos, en los que se movía Sraffa, estos temas eran objetos comunes de discusión.

La mediación de Sraffa en una posible influencia indirecta de Antonio Gramsci sobre Wittgenstein fue propuesta originalmente por Ferruccio Rossi-Landi (1970), luego de lo cual varios autores han desarrollado esa idea, por ejemplo: Alessandro Roncaglia (1980), John B. Davis (1988) y Amartya Sen (2003, p. 1241). Rossi-Landi (1970) plantea que Wittgenstein identificó a partir de Gramsci la dimensión social del lenguaje, es decir, la relación entre el lenguaje y la cultura, a partir de lo cual propone una filosofía del lenguaje que se pregunta por la creación de significados, la cual está atada a las formas de vida (Mancuso, 2010, pp. 247-248).

La amistad de Sraffa con Gramsci fue muy cercana. Sraffa fue miembro del Grupo de Estudiantes Socialistas y formó parte, desde 1919, del equipo editorial de L'Ordine Nuovo, una revista de izquierda editada por Antonio Gramsci, en la que Sraffa se encargó de la traducción al italiano de artículos en inglés, francés y alemán. También fue cercano, aunque sin ser miembro, del Partido Comunista Italiano, fundado en 1921 y liderado por Gramsci. En 1923, Gramsci deseaba confiar a Sraffa la responsabilidad de un boletín sobre los problemas laborales, titulado Senso Comune (Naldi, 2000, p. 105) ${ }^{6}$. Cuando Keynes le escribió a Sraffa comunicándole la posibilidad de vincularse a la Universidad de Cambridge como profesor (enero de 1927), Gramsci acababa de ser arrestado (noviembre 8 de 1926) y se le había impuesto una condena de 20 años. Piero Sraffa dispuso de una cuenta abierta en la librería de Milán Sperling \& Kupter para que Gramsci pudiese hacer desde su sitio de reclusión pedidos de libros, revistas y periódicos ${ }^{7}$. Durante su reclusión, entre 1929 y 1935, Gramsci escribió los 32 Cuadernos de la cárcel.

6 Nerio Naldi ha estudiado la relación entre Sraffa y el líder comunista italiano entre 1919 y 1927. 
El Cuaderno N. ${ }^{\circ} 11$, escrito entre 1932 y 1933, es el esbozo de una Introducción al estudio de la filosofía (Gramsci, 1985). Allí se plantea la existencia de una filosofía espontánea:

Por tanto, hay que demostrar preliminarmente que todos los hombres son 'filósofos', definiendo los límites y los caracteres de esa 'filosofía espontánea', propia de 'todo el mundo', esto es de la filosofía que está contenida: 1) en el lenguaje mismo, que es un conjunto de nociones y de conceptos determinados y no solamente de palabras gramaticalmente vacías de contenido; 2) en el sentido común y buen sentido; 3 ) en la religión popular y por lo tanto en todo el sistema de creencias, supersticiones, opiniones, modos de ver y actuar que se revelan en aquello que generalmente se llama 'folklore'. (Gramsci, 1975, p. 245)

Gramsci plantea que todo individuo es filósofo y que el lenguaje es una forma elemental de filosofia; el lenguaje es un objeto de estudio privilegiado: "Incluso en la mínima manifestación de una actividad intelectual, el 'lenguaje', se halla contenida una determinada concepción del mundo" (Gramsci, 1975, p. 245). El interés de Gramsci por la lingüística data de su formación juvenil en la Universidad de Turín, en el año académico 1910-1911; allí el joven sardo fue alumno de Matteo Giulio Bartolí, quién desarrolló la teoría de la "lingüística espacial", una escuela que estuvo atenta a lo dialectológico y a la heterogeneidad lingüística, de cuyo contacto surge un bloque lingüístico relativamente estable del que es posible desglosar una variedad dominante y varias dominadas, cuyas huellas están presentes en forma de sustrato (Bentivegna, 2013, pp. 11-16).

Gramsci relaciona explícitamente el estudio del lenguaje con el de la política, la cultura, la filosofía y el sentido común (Ives, 2004, p. 89). La concepción del mundo, las formas de pensar y de actuar, que compartidas definen la pertenencia a un grupo social, implican la importancia de las reglas y convenciones. En el caso particular del lenguaje, si el significado de una palabra está en su uso, deben existir reglas que gobiernen dicho uso, reglas comunes que responden a criterios socialmente aceptados (Rossi-Landi, 1970, p. 107), lo cual se encuentra, en términos similares, en la idea wittgensteriana de los juegos del lenguaje y en lo que se ha llamado la vía antropológica propuesta por Gramsci:

7 Véanse las cartas de Gramsci a Sraffa del 11 y del 21 de diciembre de 1926 (Gramsci, 2003, pp. 42, 47-48). 
Por la propia concepción del mundo se pertenece siempre a un determinado agrupamiento, y precisamente a aquel de todos los elementos sociales que comparten un mismo modo de pensar y de actuar. Se es conformista de cierto conformismo, se es siempre hombre-masa u hombres-colectivos. La cuestión es ésta: ¿de qué tipo histórico es el conformismo, el hombre-masa del que se forma parte? (Gramsci, 1975, p. 246)

La gramática para Gramsci es un objeto en construcción, es el producto de una elaboración histórica que tiene una forma social manifiestamente política, en la medida en que implica formas prescriptivas que generan formas orales y escritas. Se trata de una "gramática normativa" que en su componente escrito incluye gramáticas, vocabularios y diccionarios; instrumentos que no son suficientes para alcanzar a convertirse en norma hegemónica, pues también requiere de un componente de instancias gramaticales normativas no escritas en la construcción de lo que se considera legítimo, tal es el caso, por ejemplo, de la prensa, las manifestaciones políticas y religiosas, la literatura, el periodismo y el cine (Bentivegna, 2013, pp. 44-45). Así, la "norma" es producto de la interacción de las políticas explícitas del Estado con el conjunto de lo social.
Las diferencias lingüísticas y la "estandarización del italiano" son preocupaciones centrales en las discusiones políticas de la época (Ives, 2004, p. 88). En 1934, año en que Gramsci redacta las notas finales, se prohíbe el uso de palabras extranjeras en los diarios italianos, se produce una tendencia a legitimar el italiano nacional por encima de las variedades regionales, que son objeto de un ataque violento por parte del propio Mussolini desde principios de la década de 1930 (Bentivegna, 2013, p. 37).

De esta manera, la convención lingüística no es neutral, es dependiente de los intereses del poder hegemónico; y la propia concepción del mundo responde a problemas planteados por la realidad concreta, cotidiana (Gramsci, 1985, p. 41). El retorno a lo cotidiano es una característica esencial del pensamiento del Wittgenstein de las Investigaciones Filosóficas (Hadot, 2007, p. 83), enfoque que es cercano al proyecto antropológico del estudio del lenguaje como acción social; Wittgenstein se empeñó en utilizar un método interpretativo para ubicar las palabras sueltas dentro de un conjunto más amplio de "juegos del lenguaje", es decir, el estudio de las formas del lenguaje como actividades o prácticas culturales que deben entenderse dentro del contexto de una comunidad de usuarios, lo que implica no preguntar por el significado de algo, 
sino fijarse en su uso (Duranti, 2000, pp. 297 y 315 ).

\section{CONCLUSIONES}

Ludwig Wittgenstein, en lo referente a la transformación de su visión filosófica, pasó de la especificación de un lenguaje ideal en el que las proposiciones del lenguaje están ordenadas de un modo completamente lógico (2015, p. 122) y con la tendencia a pensar que se trata de una construcción colectiva universal de un lenguaje ideal capaz de representar el mundo, en la que el lenguaje y el mundo que este describe comparten una estructura lógica común, una proposición es una imagen de la realidad que describe, independientemente de las circunstancias sociales en las cuales el lenguaje es empleado, a la búsqueda de las reglas del lenguaje en su uso: "el significado de una palabra es su uso en el lenguaje" (2009, p. 205), en la que los sentidos se acumulan a través de una constante reorganización, en la que las convenciones $\mathrm{y}$ reglas sociales proporcionan significados particulares a las expresiones.

Keynes, Ramsey y Sraffa tuvieron una influencia importante en la evolución del pensamiento de Wittgenstein, él así lo reconoció, aunque resulta imposible especificar la naturaleza de dicho influjo. Fue Sraffa, un conocedor y crítico de las proposiciones atómicas propias de la teoría económica ortodoxa, quien presuntamente convenció a Wittgenstein de la importancia de adoptar una perspectiva antropológica para abordar los problemas filosóficos. El gesto napolitano lo pudo haber invitado a abandonar la teoría figurativa del lenguaje y a aceptar como signo algo que no respondía a las reglas de la gramática. El gesto napolitano solo se puede entender a partir de las reglas $\mathrm{y}$ convenciones establecidas en el juego del lenguaje propio del mundo napolitano, característico de su uso en un contexto social particular.

La incorporación de la perspectiva "antropológica" en el método filosófico de Wittgenstein posiblemente proviene, con la intermediación de Sraffa, de Antonio Gramsci. Tanto en la filosofía del segundo Wittgenstein, el de las Investigaciones Filosóficas, como en el proyecto filosófico de Gramsci, en las situaciones lingüísticas cotidianas la comunicación funciona porque se sobreentiende un acuerdo simbólico común entre quienes participan de un mismo juego del lenguaje.

\section{AGRADECIMIENTOS}

El presente artículo hace parte del proyecto de investigación Lógicas sociales de la moneda: un análisis multidisciplinario, el cual es financiado por la Universidad Central. Una versión preliminar de este trabajo se presentó en la Sexta Conferencia Bi-anual de la Asociación Latinoamericana de 
Historia del Pensamiento Económico, actividad que se realizó en Bogotá entre el 29 de noviembre y el 1 de diciembre de 2017. El autor agradece los comentarios y sugerencias de los evaluadores anónimos. Así mismo, asume la responsabilidad por las opiniones contenidas en el presente artículo.

\section{REFERENCIAS}

Backhouse, R. E. \& Bateman, B. W. (2014). John Maynard Keynes: un capitalista revolucionario. México, D.F.: Fondo de Cultura Económica.

Bentivegna, D. (2013). Un arcángel devastador: Gramsci, las lenguas, la hegemonía. En A. Gramsci, Escritos sobre el lenguaje (pp. 11-50). Buenos Aires: Editorial de la Universidad Nacional Tres de Febrero.

Bouveresse, J. (2006). Wittgenstein: la modernidad, el progreso, la decadencia. J. C. González \& M. M. Valdés (trads.). México, D.F.: Universidad Nacional Autónoma de México.

Davis, J. B. (1988, marzo). Sraffa, Wittgenstein and Neoclassical Economics. Cambridge Journal of Economics, 12(1), 29-36. doi:http://dx.doi.org/10.1093/oxfordjournals. cje.a035046

Dostaler, G. (2007). Keynes and his battles. Northampton, MA, USA: Edward Elgar.

Duranti, A. (2000). Antropología lingüística. P. Tena (trad.) Madrid: Cambridge University Press.

Fann, K. T. (1975). El concepto de filosofía en Wittgenstein. M. A. Beltrán (trad.). Madrid: Tecnos.

Gramsci, A. (1975). Cuaderno 4 (XIII) 1930-1932. Apuntes de filosofía I. Miscelánea. El canto décimo del Infierno. En A. Gramsci, Cuadernos de la cárcel (pp. 129-244). México, D.F.: Era, Benemérita Universidad Autónoma de Puebla.

Gramsci, A. (1985). Introducción al estudio de la filosofía. Barcelona: Crítica.

Gramsci, A. (2003). Cartas de la cárcel: 1926-1937. D. Kanoussi \& C. Ortega Kanoussi (trad.). México, D.F.: Era, Benemérita Universidad Autónoma de Puebla y Fundazione Istituto Gramsci.

Hadot, P. (2007). Wittgenstein y los límites del lenguaje. M. Arranz (trad.). Valencia, España: Pre-Textos.

Ives, P. (2004). Lenguaje, acción y hegemonía. Una propuesta gramsciana al posmarxismo. En D. Kanoussi (ed.), Poder y hegemonía hoy: Gramsci en la era global (pp. 79-95). México, D.F.: Benemérita Universidad Autónoma de Puebla. 
Malcolm, N. (2001). A memoir. En N. Malcolm, Ludwing Wittgenstein. A Memoir (pp. 21-84). Oxford: Oxford University Press.

Mancuso, H. R. (2010). De lo decible. Entre semiótica y filosofia: Pierce, Gramsci, Wittgenstein. Buenos Aires: Sb.

McGuinness, B. (2008). Wittgenstein in Cambridge: Letters and Documents 1911-1951. Melbourne, Australia: Blackwell.

Monk, R. (1994). Ludwig Wittgenstein. El deber de un genio. D. Alou (trad.). Barcelona: Anagrama.

Naldi, N. (2000). The Friendship between Piero Sraffa and Antonio Gramsci in the Years 1919-1927. European Journal of the History of Economic Thought, 7(1), 79-114. doi:http://dx.doi.org/10.1080/096725600361861

Peña, J. I. (1994). Wittgenstein y la crítica de la racionalidad. Bogotá: Universidad Nacional de Colombia, Ecoe.

Ramsey, F. P. (1923, oct.). Critical notices: «Tractatus Logico-Philosophicus». By Ludwig Wittgenstein. Mind, 32(128), 465-478.

Ramsey, F. P. (2005). Recensión crítica del Tractatus de Wittgenstein (1923). En F. P. Ramsey, Obra filosófica completa (pp. 61-82). Granada, España: Comares.

Roncaglia, A. (1980). Sraffa y la teoría de los precios. Madrid: Pirámide.

Roncaglia, A. (2006). La riqueza de las ideas: una historia del pensamiento económico. J. P. Escutia (trad.). Zaragoza, España: Prensas Universitarias de Zaragoza.

Rossi-Landi, F. (1966/1970). Por un uso marxista de Wittgenstein. En F. Rossi-Landi, El lenguaje como trabajo y como mercado (pp. 93-149). Caracas: Monte Ávila.

Sen, A. (2003, dic.). Sraffa, Wittgenstein, and Gramsci. Journal of Economic Literature, 41(4), 1240-1255. doi:http://dx.doi.org/10.1257/jel.41.4.1240

Sraffa, P. (1983). Producción de mercancías por medio de mercancias: preludio a una crítica de la teoría económica. L. A. Rojo (trad.) Barcelona: Oikos-Tau.

Wittgenstein, L. (2015). Tractatus logico-philosophicus. J. Muñoz \& I. Reguera (trads.) Madrid: Alianza.

Wittgenstein, L. (2009). Wittgenstein I. En L. Wittgenstein, Investigaciones filosóficas (pp. 155-633). Madrid: Gredos.

Wright, G. H. (2001). A Biographical Sketch. En N. Malcolm, Ludwing Wittgenstein. A Memoir (pp. 1-20). Oxford: Oxford University Press. 